EPJ Web of Conferences 84, 01001 (2015)

DOI: $10.1051 /$ epjconf/20158401001

(C) Owned by the authors, published by EDP Sciences, 2015

\title{
The $\mathrm{HD}^{+}$dissociative recombination rate coefficient at low temperature
}

\author{
A. Wolf ${ }^{1, a}$, O. Novotný ${ }^{2}$, H. Buhr ${ }^{2}$, C. Krantz ${ }^{2}$, I.F. Schneider ${ }^{3}$, O. Motapon ${ }^{4}$ and J.Zs. Mezei ${ }^{3,5}$ \\ ${ }^{1}$ Max-Planck-Institut für Kernphysik, 69117 Heidelberg, Germany \\ ${ }^{2}$ Columbia Astrophysics Laboratory, Columbia University, New York, NY 10027, USA \\ ${ }^{3}$ Laboratoire Ondes et Milieux Complexes UMR-6294 CNRS and Université du Havre, \\ 25, rue Philippe Lebon, BP. 540, 76058 Le Havre, France \\ ${ }^{4}$ LPF, UFD Mathémathiques, Informatique Appliquée et Physique Fondamentale, University of \\ Douala, PO Box 24157, Douala, Cameroun \\ ${ }^{5}$ Laboratoire Aimé Coton, CNRS-UPR-3321, Univ. Paris-Sud et Ecole Normale Supérieure de \\ Cachan, 91405 Orsay, France
}

\begin{abstract}
The effect of the rotational temperature of the ions is considered for lowenergy dissociative recombination (DR) of $\mathrm{HD}^{+}$. Merged beams measurements with $\mathrm{HD}^{+}$ ions of a rotational temperature near $300 \mathrm{~K}$ are compared to multichannel quantum defect theory calculations. The thermal DR rate coefficient for a Maxwellian electron velocity distribution is derived from the merged-beams data and compared to theoretical results for a range of rotational temperatures. Good agreement is found for the theory with $300 \mathrm{~K}$ rotational temperature. For a low-temperature plasma environment where also the rotational temperature assumes $10 \mathrm{~K}$, theory predicts a considerably higher thermal DR rate coefficient. The origin of this is traced to predicted resonant structures of the collisionenergy dependent DR cross section at few-meV collision energies for the particular case of $\mathrm{HD}^{+}$ions in the rotational ground state.
\end{abstract}

\section{Introduction}

The deuterated hydrogen molecular ion $\mathrm{HD}^{+}$serves as a prototype for studying the dissociative recombination (DR) process both experimentally [1-4] and theoretically [4, 5]. The electronic structure of the molecule has the same fundamental character as for $\mathrm{H}_{2}^{+}$. On the other hand, the substitution of one proton by a deuteron has particular advantages for the experimental study of this molecule, since it leads to its vibrational and rotational relaxation within the ground electronic state by spontaneous infrared emission [6]. In fact, the molecule thermalizes with its radiative environment at time constants on the order of $0.3 \mathrm{~s}$ for vibration and $\sim 150 \mathrm{~s}$ for rotation [7]. Thus, the storage of the $\mathrm{HD}^{+}$ion in the ultrahigh vacuum of an ion storage ring yields a vibrationally and, to a large degree, also a rotationally thermalized distribution of internal molecular states.

\footnotetext{
${ }^{a}$ Corresponding author: A. Wolf@mpi-hd .mpg.de
}

This is an Open Access article distributed under the terms of the Creative Commons Attribution License 4.0, which permits unrestricted use, distribution, and reproduction in any medium, provided the original work is properly cited. 
Using merged electron and ion beams in a storage ring, recent measurements of the $\mathrm{HD}^{+} \mathrm{DR}$ rate were performed at a resolution in the collision energy $E_{d}$ ranging down to $\sim 1 \mathrm{meV}$, yielding the energy dependent rate coefficient $\alpha_{\mathrm{mb}}\left(E_{d}\right)$. This can be compared in a detailed way to the cross section $\sigma(E)$ calculated for the process, essentially taking into account the convolution with electron energy distribution $f\left(E, E_{d}\right)$. In addition, the calculation has to account for the internal state population of the $\mathrm{HD}^{+}$ions. While the initial vibrational state is fixed to $v_{i}^{+}=0$ by the spontaneous relaxation during the ion storage, individual populations $p_{N_{i}^{+}}$must be taken into account for the various initial rotational states $N_{i}^{+}$. As a simplification, a rotational temperature $T_{\text {rot }}$ can be introduced to obtain $p_{N_{i}^{+}}$from a canonical distribution. In the theory-experiment comparison [4], then $T_{\text {rot }}$ enters as an additional parameter. The addition of the different cross section contributions $\sigma_{N_{i}^{+}}(E)$ for various $N_{i}^{+}$and the lack of detailed experimental information about the rotational population distribution, requiring simplified assumptions, increases the complexity and uncertainty in this comparison. Previous studies aiming at the resonant structures of $\sigma_{N_{i}^{+}}(E)$ at low collision energy have found a general agreement of the predicted magnitude of the DR rate coefficients, but have also shown that a direct theory-experiment comparison is still jeopardized by the superposition of several rotational contributions in the experimental data.

In the present paper, we discuss some aspects of the rotational temperature dependence of the DR rate coefficient becoming accessible through recently introduced analysis methods for the experimental data for $\alpha_{\mathrm{mb}}\left(E_{d}\right)$ and through large-scale, rotational multichannel quantum defect theory (MQDT) calculations [4] of the DR cross section $\sigma_{N_{i}}(E)$. Focusing on the magnitude of the low-temperature DR rate coefficient, we consider the Maxwellian-averaged thermal DR rate coefficient $\alpha\left(T_{e} ; T_{\text {rot }}\right)$, which assumes an isotropic thermal energy distribution at temperature $T_{e}$ for the electrons and a separate parameter $T_{\text {rot }}$ describing the ion rotational temperature. In general, the variation of $\alpha\left(T_{e}\right)$ lies close to a power-law $T_{e}^{-1 / 2}$, which is obtained when $\sigma(E)$ follows the Wigner threshold dependence $\propto E^{-1}$ predicted in the most simplified theoretical treatment. Deviations of $\alpha\left(T_{e}\right)$ from this power law describe the specific influence of individual molecular energy levels and their quantum mechanical coupling, as resulting from the detailed molecular structure.

In a recent work [8] the determination of $\alpha\left(T_{e}\right)$ from the measured merged-beams rate coefficient $\alpha_{\mathrm{mb}}\left(E_{d}\right)$ was re-considered taking into account the high-resolution energy dependence of these experimental results. The method was applied to the $\mathrm{DR}$ rate of $\mathrm{HCl}^{+}$, where it revealed significant deviations from the $T_{e}^{-1 / 2}$ power law dependence for $\alpha\left(T_{e}\right)$. Although for this system the variation of $\alpha_{\mathrm{mb}}\left(E_{d}\right)$ with $E_{d}$ was rather smooth, showing little resonant structure, the measurement was characterized by a strong drop in the recombination rate already at low energies. This resulted in a much faster decrease than $\propto T_{e}^{-1 / 2}$ in the derived $\alpha\left(T_{e}\right)$.

The merged-beams rate coefficient for $\mathrm{HD}^{+} \mathrm{DR}$ considered in the present work is dominated by strong resonant structures. The new experimental analysis method is general enough to determine the detailed $T_{e}$ dependence of $\alpha\left(T_{e}\right)$ also in this more complicated situation, based on recent highresolution data [4]. Thus, we derive experimental thermal rate coefficient curves $\alpha\left(T_{e}\right)$ in the range of low temperatures $T_{e}$ for the fundamental case of $\mathrm{HD}^{+}\left(v_{i}^{+}=0\right)$. Moreover, we compare the magnitude and the $T_{e}$ dependence with thermally averaged theoretical results. Based on these results, we point out the predicted variations of the $\mathrm{HD}^{+} \mathrm{DR}$ rate coefficient as the rotational temperature $\left(T_{\text {rot }}\right)$ lowers down to $\sim 10 \mathrm{~K}$, where only the rotational ground state remains populated.

\section{Results}

The merged beams rate coefficient measured [4] for $v_{i}^{+}=0 \mathrm{HD}^{+}$ions at the ion storage ring TSR is shown in Fig. 1. For absolute calibration, the rate coefficient measured at high impact energy, $E_{d}=9.8 \mathrm{eV}$, is matched to the absolutely measured merged-beams rate coefficient at this energy in previous experiments [3] which also demonstrates the consistence of the quantitative results from 

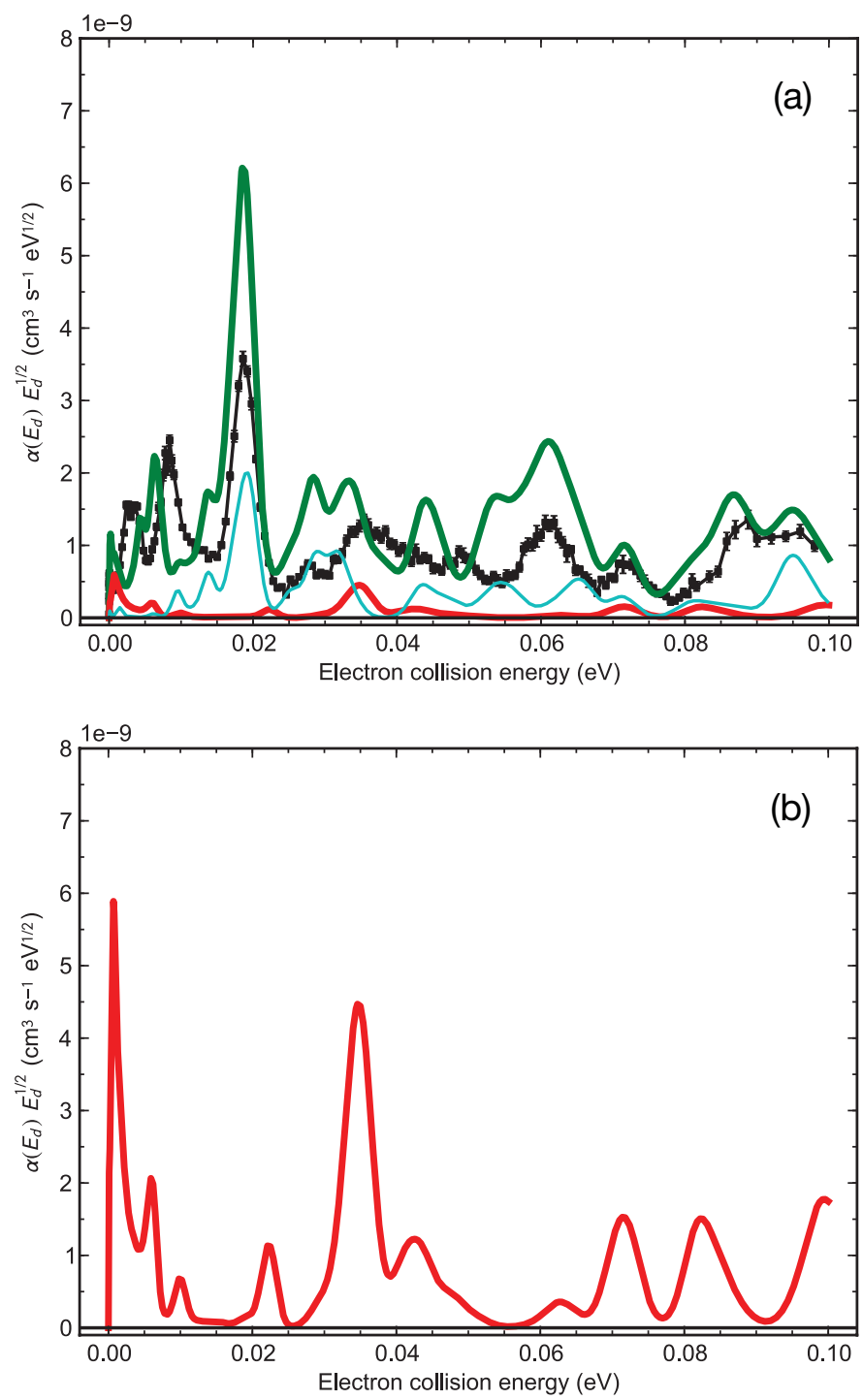

Figure 1. Scaled merged beams rate coefficient for the DR of $\mathrm{HD}^{+}$. a) Experimental data (dots) compared to theory for the thermal average at $T_{\text {rot }}=300 \mathrm{~K}$ (upper line) and for the thermal fractions at this temperature in the levels $N_{i}^{+}=0$ (lower thick line) and $N_{i}^{+}=2$ (lower thin line). The higher $N_{i}^{+}$included in the thermally averaged theory are not displayed. The electron temperatures used in the convolution of the calculated cross section are $k_{B} T_{\perp}=0.5 \mathrm{meV}$ and $k_{B} T_{\|}=0.02 \mathrm{meV}$. b) Predicted result [4] for $T_{\text {rot }}=10 \mathrm{~K}$, corresponding to essentially pure $N_{i}^{+}=0$ population.

three ion storage ring facilities. The present high-resolution rate coefficient is multiplied by $E_{d}^{1 / 2}$ to sensitively display the variations of the rate. The agreement between the experimental result and the rotationally averaged theory [Fig. 1a] has been discussed earlier [4]. For $T_{\text {rot }}=300 \mathrm{~K}$, theory succeeds in reproducing the overall magnitude and the approximate peak structure of the experiment. A separate display of the theoretical result for $T_{\text {rot }}=10 \mathrm{~K}$ [Fig. 1b] shows that the energy dependence of the 


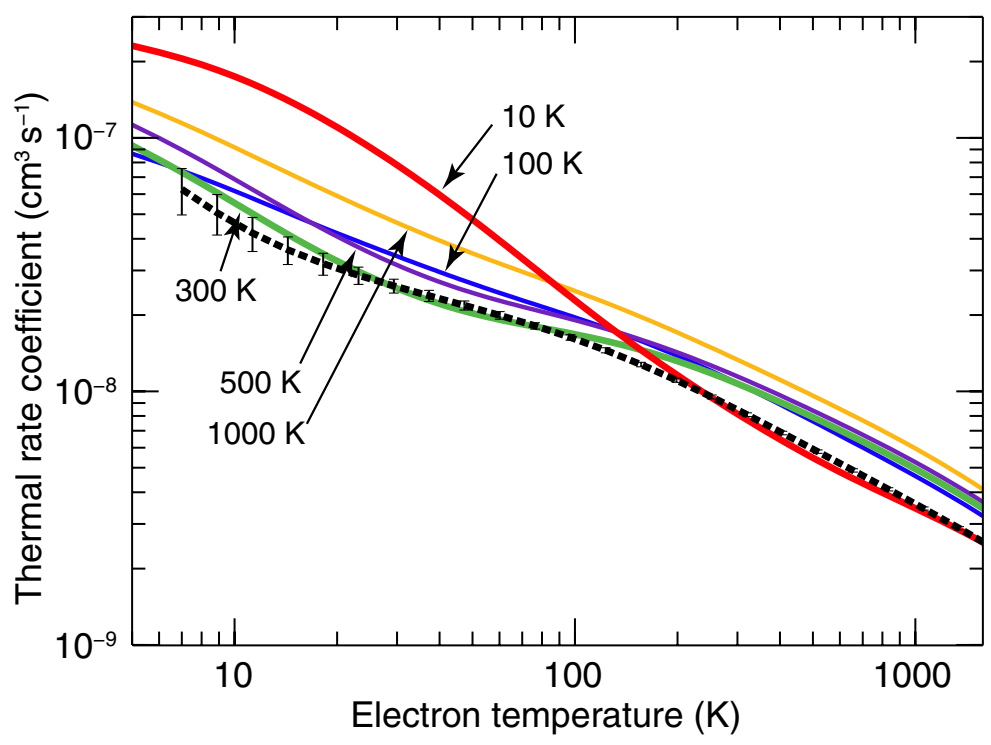

Figure 2. Thermal rate coefficient for $\mathrm{HD}^{+} \mathrm{DR}$ as a function of the electron temperature. Experiment is given by a the thick dashed line; $1 \sigma$ estimated experimental errors due to uncertainties in the electron beam temperatures are also indicated. Theory is obtained for the given rotational temperatures.

merged-beams rate coefficient is predicted to change strongly in comparison to that at $300 \mathrm{~K}$, with significant shifts in the dominant peaks of $\alpha_{\mathrm{mb}}\left(E_{d}\right)$.

The thermal rate coefficient $\alpha\left(T_{e}\right)$ is derived from these data over their full energy range up to $20 \mathrm{eV}$. Applying the recently established procedure [8] the measured $\alpha_{\mathrm{mb}}\left(E_{d}\right)$ is fitted by the sum of a large number of individual signals, each assuming a constant cross section contribution stemming from a narrow energy bin only. The energy binning for these contributions is adapted to the measured structures and the contribution magnitudes are found so that their superposition optimally fits the measured data. The free parameters used to optimize the fit are the cross section amplitudes in each energy bin. The cross section function $\hat{\sigma}(E)$ is then used to derive the Maxwellian average $\alpha\left(T_{e}\right)$. This thermal rate coefficient is shown in Fig. 2. For comparison, thermal rate coefficients $\alpha\left(T_{e} ; T_{\text {rot }}\right)$ have been deduced from the theoretical DR cross sections of $\mathrm{HD}^{+}\left(v_{i}^{+}=0, N_{i}^{+}=0, \ldots, 12\right)$ obtained by MQDT calculations [4]. Canonical averages of these $N_{i}^{+}$contributions were obtained for some values of $T_{\text {rot }}$ between $1000 \mathrm{~K}$ and $10 \mathrm{~K}$.

The rotational level populations expected for the storage ring experiment underlying the measured data in Fig. 2 are estimated to be close to a 300-K thermal distribution [4], with a likelihood of somewhat lower effective $T_{\text {rot }}$ (down to $\sim 150 \mathrm{~K}$ ) because of superelastic cooling of the rotational $\mathrm{HD}^{+}$levels by collisons in the cold merged electron beam $[4,9]$. Considering the variation of $\alpha\left(T_{e} ; T_{\text {rot }}\right)$ with the rotational temperature, the theoretical prediction for $T_{\text {rot }}=300 \mathrm{~K}$ in fact yields a very good overall agreement with the measurement. The prediction lies near the measured data over the full temperature range and in particular very closely reproduces their magnitude in the low-temperature range $T_{e}<$ $100 \mathrm{~K}$. The theoretical result for $T_{\text {rot }}=200 \mathrm{~K}$ is nearly indistinguishable from that at $T_{\text {rot }}=300 \mathrm{~K}$. At $T_{\text {rot }}=500 \mathrm{~K}$ theory better reproduces the experimental variation of $\alpha\left(T_{e}\right)$ if the experimental rates are scaled up by a factor of $\sim 1.2$. However, the absolute deviation of $\sim 20 \%$ somewhat exceeds the expected systematic error of the experiment.

A significant increase of the thermal rate coefficient in the low-temperature range, amounting to more than a factor of 2 , is predicted by theory when the rotational temperature of the $\mathrm{HD}^{+}$ions is 
reduced to $10 \mathrm{~K}$. This corresponds to more than $99.4 \%$ of all ions in the state $N_{i}^{+}=0$. While this prediction has not yet been verified experimentally, it shows that the $\mathrm{DR}$ rate of $\mathrm{HD}^{+}$ions in a cold environment with $T_{\text {rot }}$ near $10 \mathrm{~K}$ is expected to be substantially different from the results presently obtained at $300 \mathrm{~K}$. The fact that the theory for $T_{\text {rot }}=300 \mathrm{~K}$ agrees well with experimental results for which a similar rotational temperature is estimated, gives some support to the prediction also for the rotationally cold case. The origin of the strongly increased low-temperature DR rate for rotationally cold $\mathrm{HD}^{+}$ions is discussed in the following on the basis of the theoretical results.

\section{Discussion}

To understand the origin of the strong low-energy contribution to the DR rate coefficient for $N_{i}^{+}=0$ we consider in Fig. 3 the calculated resonant structures of the rate coefficients for two initial rotational states of $\mathrm{HD}^{+}$. Here we show the specific theoretical rate coefficient $\alpha_{t, N_{i}^{+}}(E)=(2 E / m)^{1 / 2} \sigma_{N_{i}^{+}}(E)$ as directly obtained from the MQDT calculations [4] of the cross sections $\sigma_{N_{i}^{+}}(E)$. We also show the convolution $\alpha_{\mathrm{mb}, N_{i}^{+}}\left(E_{d}\right)$ of this rate with the experimental electron energy distribution $f\left(E, E_{d}\right)$. In order to keep the plotted amplitudes of similar size along the energy scale, both rate coefficients are scaled by multiplying them with the square root of the electron collison energy, using $E$ and $E_{d}$ for $\alpha_{t}(E)$ and $\alpha_{\mathrm{mb}}\left(E_{d}\right)$, respectively.

The rate coefficients show rich resonant structure, where many of the narrow features remain unresolved at the experimental energy resolution. In order to compare the resonance positions to Rydberg series associated with rotationally or vibrationally excited $\mathrm{HD}^{+}$cores, such resonances are indicated. Here, the excitation energies from the $\left(v_{i}^{+}=0, N_{i}^{+}\right)$initial state to an excited $\mathrm{HD}^{+}$state $\left(v^{+}\right.$, $N^{+}$) are taken from earlier precision calculations [10]. From these energies, the binding energy of the captured electron in a Rydberg level $n l$ is subtracted. For purpose of displaying these series, constant quantum defects $\bar{\mu}_{l}$ are used for all $n$. The relevant electron angular momenta are $l=0$ and $l=2$, leading to $N^{+}=\max \left(0, N_{i}^{+} \pm \delta\right)$ with $\delta=0$ and 2 for $l=0$ and $\delta=0,2$ and 4 for $l=2$. Values of $\bar{\mu}_{0}=-0.0835$ and $\bar{\mu}_{2}=0.0346$ are used for $\mathrm{s}$ and $\mathrm{d}$ states, respectively. Full quantum defect functions of the internuclear distance $R$ are used in the MQDT calculations [4], together with suitable functions describing the coupling between the various Rydberg channels as a function of $R$. The Rydberg series shown are restricted to those where levels appear in the range of low electron collision energies.

In the theoretical rate coefficient spectra before convolution, broader structures appear to be related to lower $n$ and correspondingly higher excitations. In particular for lower $n$ values, also significant shifts are observed from the resonance positions obtained in the simple constant $\bar{\mu}_{l}$ calculation. We compare the rate coefficient for the initial $\mathrm{HD}^{+}$level $N_{i}^{+}=0$ with that of $\mathrm{HD}^{+}$in $N_{i}^{+}=2$, which yields one of the most important contributions to the rotationally averaged rate coefficient at $T_{\text {rot }}=300 \mathrm{~K}$ [see Fig. 1a]. The $N_{i}^{+}=0$ calculation clearly shows a broad structure at very low collison energy, ranging down to $1 \mathrm{meV}$ or below, as well as several low-lying Rydberg resonances for the excited core states $N^{+}=2$ and $N^{+}=4$. The $n=16$ resonance of the $\left(v^{+}=0, N^{+}=4\right)$ core, connected to purely rotational excitation and a relatively low $n$, is nearly degenerate with the lowest HD ionization threshold and thus lies at sub-meV electron collision energies for $\mathrm{HD}^{+}\left(v_{i}^{+}=0, N_{i}^{+}=0\right)$ ions. Moreover, a broad resonance, possibly related to the $3 \mathrm{~s}$ level for vibrational excitation to $v^{+}=7$, occurs at similar energies and boosts the DR rate coefficient at very low collision energies. Taking into account the scaling of $\alpha_{t}(E)$ and $\alpha_{\mathrm{mb}}\left(E_{d}\right)$ used in Fig. 3, the averaged merged-beams rate coefficient for $\mathrm{HD}^{+}\left(v_{i}^{+}=0, N_{i}^{+}=0\right)$ ions (corresponding to $T_{\text {rot }} \sim 10 \mathrm{~K}$ ) amounts to $\sim 1.5 \times 10^{-7} \mathrm{~cm}^{3} \mathrm{~s}^{-1}$ at a collision energy of $E_{d}=1 \mathrm{meV}$, about a factor of 2 higher than that presently observed in the high-resolution measurement for $\mathrm{HD}^{+}$at $T_{\text {rot }} \sim 300 \mathrm{~K}$. In addition, the energy dependence of the $N_{i}^{+}=0$ contribution shown in Fig. 1 is strongly different from both the observation and the rotationally averaged theory for $T_{\text {rot }}=300 \mathrm{~K}$. This suggests that a strong change of the merged beams rate coefficient for this system can be expected by rotationally 

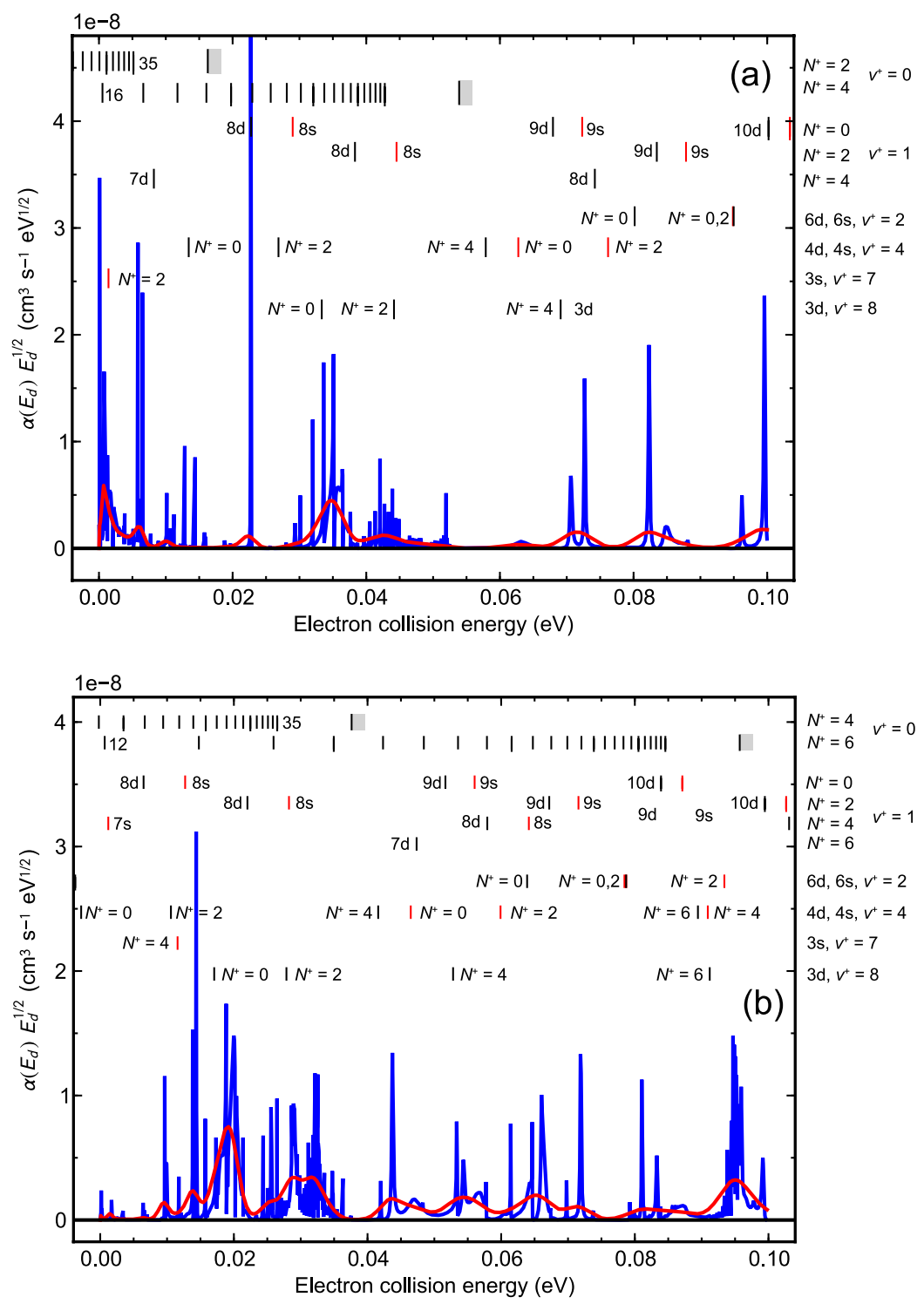

Figure 3. Theoretical scaled specific rate coefficient $\left.\alpha_{t, N_{i}^{+}}(E)\right) E^{1 / 2}$ (line with sharp structures) and the corresponding scaled merged-beams rate coefficient $\alpha_{\mathrm{mb}, N_{i}^{+}}\left(E_{d}\right) E_{d}^{1 / 2}$ resulting from the convolution with the experimental electron energy distribution $f\left(E, E_{d}\right.$ ) (smoothed line) for $N_{i}^{+}=0$ (a) and $N_{i}^{+}=2$ (b). The positions of $n \mathrm{~d}$ and $n$ s Rydberg states (calculated as discussed in the text) are represented by black and red vertical lines, respectively.

cooling the ions. Similarly, only measurements with rotationally cold ions can be expected to yield reliable DR data for low-temperature environments.

We thank the MPIK accelerator and TSR crews for their excellent assistance. ON was supported in part by the NSF Division of Astronomical Sciences Astronomy and Astrophysics Grants program and by the NASA Astronomy and Physics Research and Analysis Program. Support by the Max-Planck Society is gratefully acknowledged. 


\section{References}

[1] P. Forck, M. Grieser, D. Habs, A. Lampert, R. Repnow, D. Schwalm, A. Wolf, D. Zajfman, Phys. Rev. Lett. 70, 426 (1993)

[2] T. Tanabe, I. Katayama, H. Kamegaya, K. Chida, Y. Arakaki, T. Watanabe, M. Yoshizawa, M. Saito, Y. Haruyama, K. Hosono et al., Phys. Rev. Lett. 75, 1066 (1995)

[3] A. Al-Khalili, S. Rosén, H. Danared, A.M. Derkatch, A. Källberg, M. Larsson, A. Le Padellec, A. Neau, J. Semaniak, R. Thomas et al., Phys. Rev. A 68, 042702 (2003)

[4] F.O. Waffeu Tamo, H. Buhr, O. Motapon, S. Altevogt, V.M. Andrianarijaona, M. Grieser, L. Lammich, M. Lestinsky, M. Motsch, I. Nevo et al., Phys. Rev. A 84, 022710 (2011)

[5] I.F. Schneider, C. Strömholm, L. Carata, X. Urbain, M. Larsson, A. Suzor-Weiner, J. Phys. B: At. Mol. Opt. Phys. 30, 2687 (1997)

[6] Z. Amitay, A. Baer, M. Dahan, L. Knoll, M. Lange, J. Levin, I.F. Schneider, D. Schwalm, A. Suzor-Weiner, Z. Vager et al., Science 281, 75 (1998)

[7] Z. Amitay, D. Zajfman, P. Forck, Phys. Rev. A 50, 2304 (1994)

[8] O. Novotný, A. Becker, H. Buhr, C. Domesle, W. Geppert, M. Grieser, C. Krantz, H. Kreckel, R. Repnow, D. Schwalm et al., Astrophys. J. 777, 54 (2013)

[9] D. Shafir, S. Novotny, H. Buhr, S. Altevogt, A. Faure, M. Grieser, A.G. Harvey, O. Heber, J. Hoffmann, H. Kreckel et al., Phys. Rev. Lett. 102, 223202 (2009)

[10] G. Hunter, A.W. Yau, H.O. Pritchard, At. Data Nucl. Data Tab. 14, 11 (1974) 